\title{
Predicting the constitutive behavior of semi-solids via a direct finite element simulation: application to AA5182
}

\author{
A B Phillion ${ }^{1}$, S L Cockcroft ${ }^{2}$ and P D Lee ${ }^{3}$ \\ ${ }^{1}$ Laboratoire de Simulation des Matériaux, Ecole Polytechnique Fédérale de Lausanne, \\ Lausanne, Switzerland \\ 2 Department of Materials Engineering, University of British Columbia, Vancouver Canada \\ ${ }^{3}$ Department of Materials, Imperial College London, London, UK
}

Received 16 January 2009, in final form 3 May 2009

Published 26 June 2009

Online at stacks.iop.org/MSMSE/17/055011

\begin{abstract}
The methodology of direct finite element (FE) simulation was used to predict the semi-solid constitutive behavior of an industrially important aluminummagnesium alloy, AA5182. Model microstructures were generated that detail key features of the as-cast semi-solid: equiaxed-globular grains of random size and shape, interconnected liquid films, and pores at the triple-junctions. Based on the results of over fifty different simulations, a model-based constitutive relationship which includes the effects of the key microstructure featuresfraction solid, grain size and fraction porosity - was derived using regression analysis. This novel constitutive equation was then validated via comparison with both the FE simulations and experimental stress/strain data. Such an equation can now be used to incorporate the effects of microstructure on the bulk semi-solid flow stress within a macro- scale process model.
\end{abstract}

\section{Introduction}

The simulation of industrial solidification processes has made major advances over the past few years, due to new computational techniques and increased computing power. This has led to the development of sophisticated models for prediction of the evolution in temperature, stress/strain and defect formation with time [1-7]. The thermal models are well described using Fourier's Law, and require only a proper description of the intricate heat transfer boundary conditions. In contrast, the stress/strain models are much more challenging since there is currently no consistent phenomenological relationship to describe the development of stresses and strains during solidification. Many studies (see review by Eskin et al [8]) have previously shown that as-cast microstructural features such as grain size and shape, porosity, intermetallics and the configuration of the liquid add to the complexity of semi-solid deformation. However, these features are difficult to experimentally control during solidification while accurately measuring constitutive behavior. 
Over the years, a number of constitutive relationships have been developed to characterize the semi-solid behavior based on experimental data. Some researchers [9-14] proposed semi-solid constitutive models based on a modified creep law where the load carrying area is related to the fraction solid or to the proportion of grain boundaries covered by liquid films. Other researchers [3,15-19] proposed two-phase models consisting of a porous solid skeleton saturated with liquid. While a number of sophisticated details have been included in these models, such as the partial cohesion parameter devised by Martin et al [15] to deal with the increased dendrite interlocking with increasing fraction solid, they remain largely empirical. Furthermore, these models have also assumed that the semi-solid stress-strain behavior is dependent only on temperature and strain rate, but is independent of microstructure features and defects. Along with the above modeling challenges, model validation is difficult since good apparatuses do not yet exist which are able to test material with a microstructure representative of industrial castings across a wide range of fraction solids. Simply cutting specimens from as-cast products, and then reheating them into the semi-solid regime is not sufficient for fraction solids less than $\sim 0.95$ since the fraction solid-temperature relationship during solidification will be different as compared with remelting, and there is no liquid feeding to counteract deformation [20]. Moreover, recent research has shown that there can be considerable differences between the solidification and partial remelting microstructures since the initial liquid during remelting will tend to form as eutectic pockets while the final liquid during solidification will tend to form as interdendritic eutectic films [12].

In other research fields in which the macroscopic mechanical properties are greatly dependent on a composite microstructure, the link between the micro and macro levels has been derived via a direct finite element (FE) simulation of a representative elementary volume instead of using experimental data. Gall et al [21] investigated fatigue in cast aluminum alloys using a microstructure/FE model with idealized geometry to show that debonded Si particles and porosity can play a major role in fatigue crack formation. Gao et al [22] also investigated fatigue in cast aluminum alloys, but used realistic geometry in combination with a validated model to predict fatigue damage limit. They also showed that the specific location of pores is critical for fatigue performance. McDowell et al [23] developed a model to examine the role of microplasticity around debonded particles and shrinkage pores in the formation of fatigue cracks. Horstemeyer et al [24] used FE simulations to determine the influence of different microstructure parameters on pore/crack nucleation in a cast A356 aluminum alloy. Pardoen and co-workers [25-28] have also developed a number of direct FE simulations, for prediction of constitutive behavior and ductile yielding under varying microstructure and alloy conditions. Harnish et al combined a simulation of the hot rolling process with a polycrystalline plasticity code in order to examine texture evolution in 705X aluminum alloys [29]. Based on these works, it is clear that microstructure modeling can be successfully used to predict constitutive behavior.

To date, the application of microstructure modeling to predict semi-solid constitutive behavior has not been possible, due to the difficulty in realistically characterizing the semisolid microstructure. However, in work by Mathier et al [30], and subsequently by Vernède et al [31,32], this difficulty was addressed through the application of a granular model using the Voronoi tessellation technique to model the solidification of globular grains. Solidification is carried out by advancing the grain edges along a linear segment connecting the nuclei with a Voronoi vertex. Phillion et al [33] then applied this Voronoi tessellation technique to demonstrate the feasibility of applying microstructure deformation models to semi-solid materials. This paper extends the technique presented in [33] in two important ways: (i) the FE model has been applied to a wide range of semi-solid microstructural conditions over which tensile deformation experiments are not viable; and (ii) a constitutive relationship is developed 


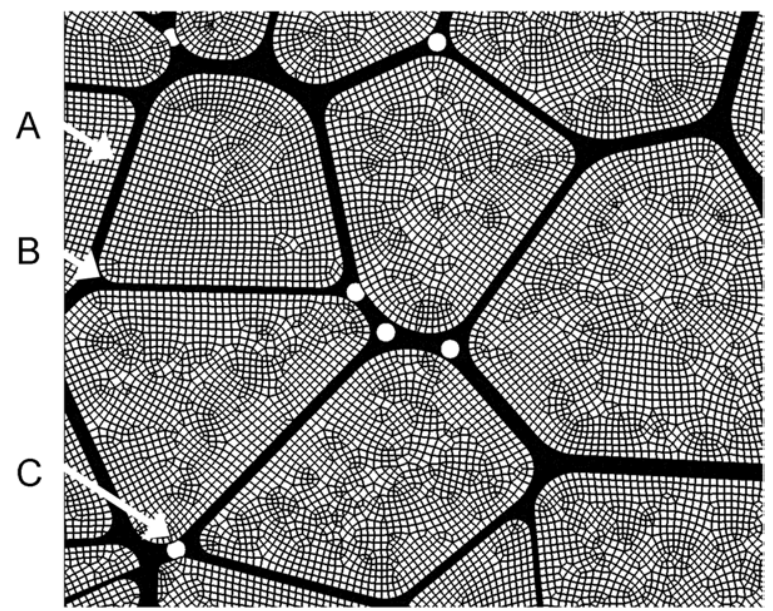

Figure 1. Critical features of the three-phase semi-solid microstructure model: A-grain boundary liquid; $\mathrm{B}$-rounded grain vertices; $\mathrm{C}$-triple-junction porosity. (Note that the mesh has been removed from the liquid for clarity).

for semi-solid materials which takes into account grain size and solidification defects. To the authors' understanding, this is the first constitutive relationship developed for semi-solids which takes into account these solidification phenomena.

In the first part of the paper, we briefly review the technique for simulating the semi-solid constitutive behavior using a direct FE model. In the second part, the stress/strain predictions made by the FE simulation as a result of grain size, as-cast porosity and fraction solid variations are discussed and analyzed. In the third part, a microstructure-based constitutive relationship is developed for a semi-solid aluminum-magnesium alloy, AA5182. Finally, the last part provides a validation of the constitutive relationship via tensile deformation experiments at high fraction solid.

\section{Model theory}

The methodology for implementation of the direct FE simulations to semi-solid materials is presented below, and builds on aspects of the theory presented in a prior publication [33].

\subsection{Model geometry}

The model geometry, a subsection of which is shown in figure 1, is derived from a $2 \mathrm{D}$ Voronoi tessellation. A Voronoi tessellation consists of a domain divided into a subset of polygonal regions, the boundaries of which are the perpendicular bisectors of lines joining a set of $N$ randomly placed discrete points. Each Voronoi region simulates a grain, with the discrete points representing grain nuclei. In this work, the representative elementary volume consists of 56 Voronoi regions on a square domain. To create the semi-solid microstructure, the Voronoi regions are reduced in size via a simple scaling law; the resulting extra material surrounding each region is assumed to be a liquid (marker ' $A$ ' in figure 1) [30]. The sharp vertices of the Voronoi regions are then rounded based on a solute flux balance between the Gibbs-Thomson effect and enhanced solute diffusion at the corners, as proposed by Vernède et al [31,32] (marker ' $B$ ' in figure 1). Removing solid material from 
Table 1. Comprehensive series of model runs conducted to predict the semi-solid constitutive behavior of AA5182.

\begin{tabular}{cll}
\hline Grain size & Fraction solid $\left(f_{\mathrm{s}}\right)$ & Fraction solid $\left(f_{\mathrm{p}}\right)$ \\
\hline 75 & $0.75,0.80,0.85,0.90,0.95,0.97$ & 0 \\
150 & $0.75,0.80,0.85,0.90,0.95,0.97^{\mathrm{a}}$ & $0,0.002,0.004,0.006$ \\
225 & $0.75,0.80,0.85,0.90,0.95,0.97^{\mathrm{a}}$ & $0,0.002,0.004,0.006$ \\
300 & $0.75,0.80,0.85,0.90,0.95,0.97$ & 0 \\
\hline
\end{tabular}

${ }^{a}$ Note that this value of $f_{\mathrm{s}}$ was only used when $f_{\mathrm{p}}=0$.

the corners has a secondary effect whereby liquid concentrates at the corners, simulating triple-junctions. Individual pores of radius $7.5 \mu \mathrm{m}$ are also randomly added to these triplejunctions to approximate the effect of as-cast porosity on semi-solid behavior (marker ' $C$ ' in figure 1).

\subsection{FE simulation}

The mesh generation and FE analysis are performed using the software package ABAQUS ${ }^{T M}$. The flow stress of the solid grains is both strain rate and temperature dependent, and is based on a Ludwik equation for semi-solid AA5182 [33], while the flow stress in the liquid is assumed to be elastic perfectly plastic with a yield stress of $0.5 \mathrm{MPa}$. The elastic modulus and Poisson's ratio of the solid grains was assumed to be $E=70 \mathrm{GPa}$ and $v=0.30$ respectively; i.e. the behavior of a low-temperature aluminum alloy. In the liquid, a small elastic modulus $(0.7 \mathrm{GPa})$ was chosen in order to offer little resistance to stress. The Poisson's ratio for the liquid was chosen to be $v=0.45$, a compromise between conserving liquid volume and minimizing numerical issues in a FE analysis. The semi-solid constitutive behavior predictions (i.e. the bulk semi-solid true stress curves as a function of strain) have been calculated based on the force required to displace the top side of the square at a bulk strain rate of $0.0015 \mathrm{~s}^{-1}$, with symmetry boundary conditions used on both the left and bottom sides. A weak point with the FE simulations may be that the effects of bulk solid grain rearrangement and fluid flow within the liquid channels cannot be fully considered. In the case of grain movement, some small scale displacement related to liquid channel deformation can be accommodated in this formulation; however, large scale movement is not. Large scale grain rearrangement can be ignored since the FE model will only be applied to the high fraction solid regime $\left(f_{\mathrm{s}}>0.75\right)$, where the configuration of the mushy zone does not permit bulk grain movement [34]. Similarly, some small scale fluid displacement can be accommodated, but not bulk flow such as may be required to accommodate large intergranular channel deformation or the volumetric shrinkage associated with solidification [35].

\section{Results and discussion}

\subsection{Direct FE simulation of semi-solids}

The three-phase microstructure model has been used to predict the constitutive behavior of semi-solid AA5182 over a range of fraction solid $\left(0.75<f_{\mathrm{s}}<0.97\right)$, fraction porosity $\left(0<f_{\mathrm{p}}<0.006\right)$ and grain size $(75 \mu \mathrm{m}<\bar{d}<300 \mu \mathrm{m} ; \bar{d}$ is the average grain diameter assuming circular grains) via direct FE simulation. A summary of the values chosen for $f_{\mathrm{s}}, f_{\mathrm{p}}$ and $\bar{d}$ are provided in table 1 . In total, 50 different stress-strain predictions from the model runs were acquired, corresponding to 50 different microstructure 


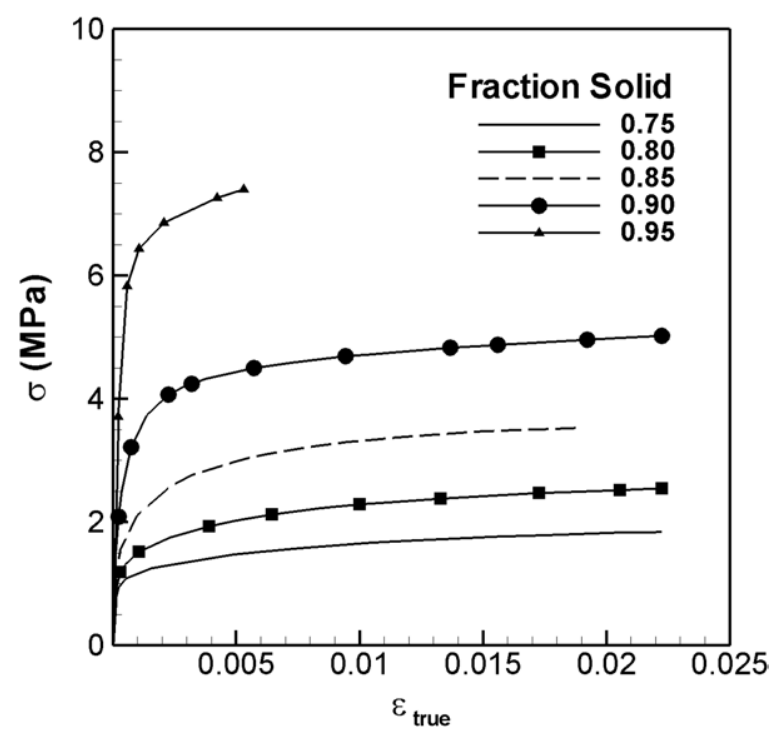

Figure 2. Effect of fraction solid on the predicted semi-solid constitutive behavior in the range $0.75<f_{\mathrm{s}}<0.95\left(\bar{d}=150 \mu \mathrm{m}, f_{\mathrm{p}}=0\right)$.

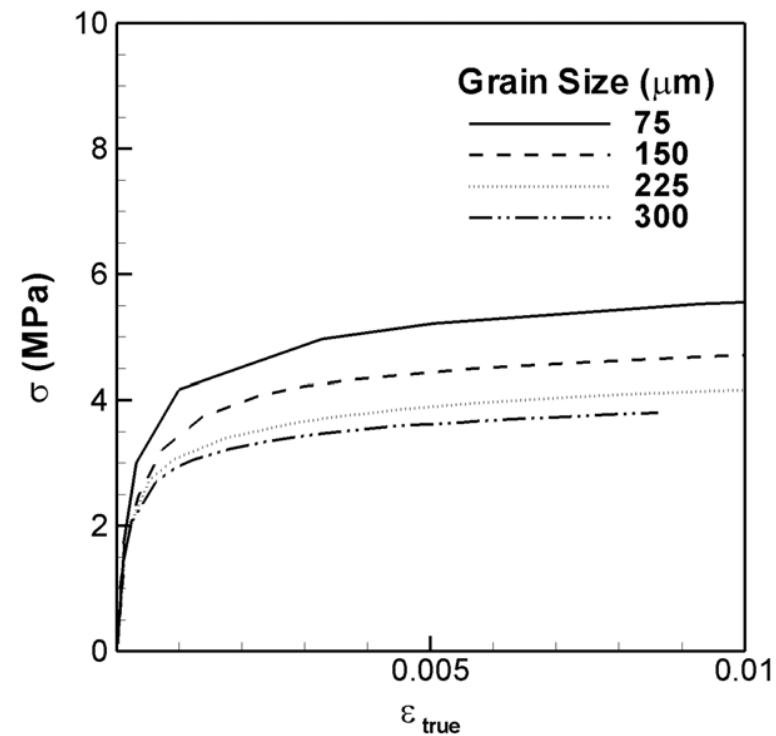

Figure 3. Effect of grain size on the predicted semi-solid constitutive behavior in the range $75<\bar{d}<300 \mu \mathrm{m}\left(f_{\mathrm{s}}=0.90, f_{\mathrm{p}}=0\right)$.

configurations. Note that the effect of porosity was investigated only at grain sizes of 150 and $225 \mu \mathrm{m}$.

The true stress-true strain predictions obtained from the comprehensive series of model runs are shown in figures 2-4. It is clear from the figures that both fraction solid and grain size have a significant effect on constitutive behavior, while the effect of fraction porosity in the range examined is moderate. 


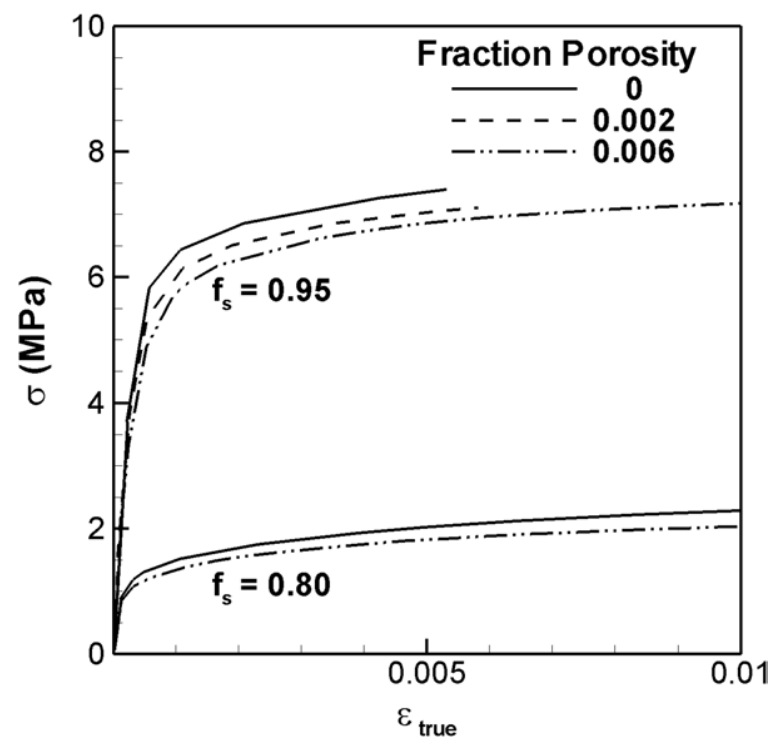

Figure 4. Effect of fraction porosity on the predicted semi-solid constitutive behavior at $f_{\mathrm{s}}=0.80$ and $f_{\mathrm{s}}=0.95(\bar{d}=150 \mu \mathrm{m})$.

Table 2. Variation in $f_{\mathrm{s}}(\mathrm{T})$ for AA5182 after Thompson [36].

\begin{tabular}{ll}
\hline Fraction solid & Temperature \\
\hline 1.0 & 525 \\
0.97 & 550 \\
0.95 & 561 \\
0.90 & 578 \\
0.85 & 588 \\
0.80 & 596 \\
0.75 & 602 \\
\hline
\end{tabular}

Fraction solid. The effect of fraction solid $\left(f_{\mathrm{s}}=0.75,0.80,0.85,0.90\right.$ and 0.95$)$ on the predicted stress-strain behavior is shown in figure 2 for the case with $\bar{d}=150 \mu \mathrm{m}$. As can be seen from the figure, the bulk semi-solid true stress decreases with decreasing fraction solid. Fraction solid was found to be the most critical of the three variables examined in this study, resulting in a $\sim 75 \%$ decrease in flow stress with a $20 \%$ decrease in the fraction solid (from $f_{\mathrm{s}}=0.95$ to $f_{\mathrm{s}}=0.75$ ). This stress decrease is related to changes occurring in both the solid grains and the liquid film. Firstly, there is a decrease in the flow stress of the solid grains due to an increase in temperature. The relationship between fraction solid and temperature is provided in table 2 [36], and the constitutive behavior of the solid is as described in [33]. Secondly, there is an increase in the thickness of the liquid channels due to the decrease in the fraction solid, allowing for greater strain accumulation in the liquid before the solid grains deform. Thirdly, the larger liquid channels allow for greater solid grain movement, further delaying interaction between the solid grains. It would thus appear that both the deformation behavior of the liquid and the interactions of the solid/liquid interface are of critical importance in describing the effects of fraction solid on semi-solid constitutive behavior. Two images of the deformed mesh at a bulk strain of $\varepsilon=0.005$, for $f_{\mathrm{s}}=0.80$ and $f_{\mathrm{s}}=0.95$, are shown in figure 5 to illustrate these features. As can be seen in the figure, the strain is completely 


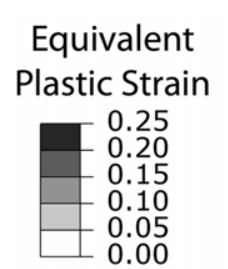

0.00

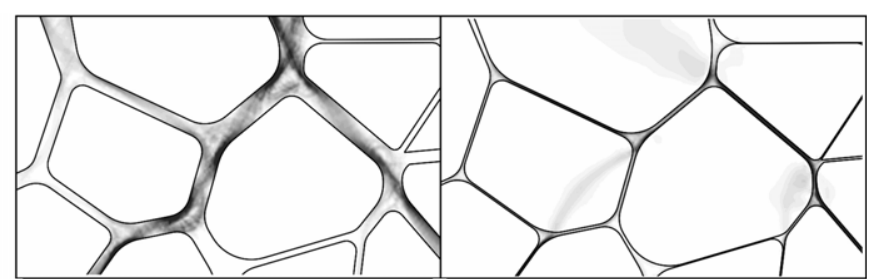

(a) fraction solid $=0.80$

(b) fraction solid $=0.95$

Figure 5. Comparison of the deformed meshes obtained from the FE simulations at $(a) f_{\mathrm{s}}=0.80$ and $(b) f_{\mathrm{s}}=0.95(\bar{d}=150 \mu \mathrm{m})$; the bulk strain $\varepsilon_{\text {bulk }}=0.005$.

accommodated in the liquid phase when $f_{\mathrm{s}}=0.80$ due to the presence of the thick liquid channels. In contrast, solid deformation and shear banding are visible in the solid grains at $f_{\mathrm{s}}=0.95$ since the liquid channels are very thin and cannot accommodate much tensile strain.

It can also be seen in figure 2 that the bulk semi-solid true stress increases with increasing strain. This hardening behavior is termed geometric strain hardening and occurs due to interaction between the solid grains. Non-classical work hardening has previously been experimentally observed in high fraction solid semi-solid aluminum alloys (AA5182 [37] and $\mathrm{Al}-2 \mathrm{wt} \% \mathrm{Cu}[4])$ under tensile loading. However, in these experiments, strain accumulation in the liquid was aided by intergranular liquid flow, which is not accounted for in the present model.

The effect of volume fraction of a second phase on the bulk constitutive behavior has also been previously examined, but within the context of metal-matrix composites (MMCs) [38-41]. However, the models developed for MMCs cannot be easily applied to the semi-solid case since the microstructural configuration, with the high-strength second phase distributed within the primary phase, is substantially different. In semi-solids, the liquid is both weaker than the solid and remains interconnected even at low volume fraction.

Grain size. The effect of grain size $(\bar{d}=75,150,225$ and $300 \mu \mathrm{m})$ is shown in figure 3 for the case with $f_{\mathrm{s}}=0.90$. As can be seen from the figure, the bulk semi-solid true stress decreases with increasing grain size. Larger grain sizes weaken the semi-solid since they result in thicker liquid channels for a given fraction solid, allowing for increased strain accumulation in the liquid before the solid grains deform. In fully solid materials, the grain size dependence is often experimentally characterized using the Hall-Petch equation. This equation has been reproduced using mono- and two-phase micro-models (e.g. Venkatramani et al [42]). However, the grain size dependence for semi-solid materials shown in figure 3 results in a Hall-Petch exponent of 0.25 , instead of 0.5 , since deformation is greatly facilitated by the interdendritic liquid [35].

Over the range of grain sizes examined with the direct FE simulations, the predicted peak flow stress decreases by $\sim 30 \%$ for a four-fold increase in grain size. Since the range of grain sizes examined in this work is large in comparison with industrially as-cast aluminum alloys, the grain size effect is shown to be of moderate importance in comparison with fraction solid. Furthermore, although not shown in figure 3, the effect of grain size decreases with decreasing fraction solid. This is because smaller values of fraction solid have thick liquid films, and changing the grain size provides only a relatively small change in film thickness. 
Table 3. Results showing the effect of grain geometric configuration at $\varepsilon=0.01$ on model predictions.

\begin{tabular}{lll}
\hline Grain size $(\mu \mathrm{m})$ & $\sigma_{\text {med }}(\mathrm{MPa})$ & $\%$ variability \\
\hline 75 & 5.52 & $15 \%$ \\
150 & 4.71 & $20 \%$ \\
225 & 4.16 & $24 \%$ \\
300 & 3.87 & $25 \%$ \\
\hline
\end{tabular}

Fraction porosity. The effect of fraction porosity on the stress-strain predictions is shown in figure 4 for the cases with $f_{\mathrm{s}}=0.80$ and 0.95 , and $\bar{d}=150 \mu \mathrm{m}$. As can be seen in the figure, the addition of porosity to the model geometry results in a modest decrease in the semi-solid bulk true stress: a $\sim 10 \%$ decrease at $f_{\mathrm{s}}=0.95$, and less than a $5 \%$ decrease for $f_{\mathrm{s}}=0.80$. This reduction in stress is related to changes within the liquid phase. Firstly, adding porosity leads to increased strain localization within the liquid, allowing deformation to occur at a decreased strain. Secondly, while the fraction porosity is quite small, this porosity acts to reduce the effective load bearing area within the model domain. Thirdly, because the pores are placed preferentially at the grain triple points, they are able to enhance grain mobility leading to a delay in grain interlocking. The effect of porosity is more pronounced at high fraction solid because the liquid films are thinner and thus the pores have a proportionally greater influence via the mechanisms highlighted above. Overall, porosity has only a small impact on the bulk semi-solid true stress, matching prior experimental results which showed that flow stress was only affected when the fraction porosity is greater than 1 or $2 \%$ [43]. However, prior experiments have shown that very small amounts of porosity can have a large impact on elongation and final failure [43], hot tearing [44] and fatigue life [45]. It is important to also point out that the current version of the model does not account for the nucleation, growth and coalescence of porosity with increasing strain. Thus, the model can only be applied within the limits of porosity examined above.

One of the interesting aspects of the model is that for specific values of $f_{\mathrm{s}}, f_{\mathrm{p}}$ and $\bar{d}$, multiple semi-solid geometries can be created because the set of points which define the grain nuclei are randomly placed within the model domain. Due to interaction between the solid and liquid, these individual geometries result in different predicted stress-strain behavior. This stochastic variability was investigated by conducting 25 model runs at $f_{\mathrm{s}}=0.90$, $f_{\mathrm{p}}=0$, for each of the four grain sizes $(75,150,225$ and $300 \mu \mathrm{m})$. The location of the grain nuclei was varied between runs providing each run with a different geometric grain configuration. The results of this analysis-the median true stress $\left(\sigma_{\mathrm{med}}\right)$ at a strain of 0.01 , and the percentage variability resulting from the 25 different model runs for all four grain sizes-are shown in table 3. As can be seen in this table, geometric configuration can have a significant effect on semi-solid constitutive behavior, especially at the larger grain sizes with a variability of $25 \%$ for a grain size of $300 \mu \mathrm{m}$. The larger grain sizes are more vulnerable to geometric configuration variation because the liquid channels are thicker, which provides for more variability in the interaction between the solid and liquid components.

In summary, the above parametric study clearly demonstrates that the microstructure model is able to predict the fine nuances of the interactions between grain size, fraction solid, fraction porosity and constitutive behavior. For example, it is predicted that at low fraction solid, porosity is not important but at high fraction solid, the location of the porosity is critical for simulation convergence and hence for the bulk stress-strain behavior. 


\subsection{Derivation of model-based constitutive equation}

The results from the direct FE simulation of semi-solid tensile deformation provide a method for quantitatively determining the effects of microstructural features on the semi-solid constitutive behavior of AA5182. However, this model is not suitable for implementation into a macroscale model since it requires length scales of the order of the grain size. To be computationally tractable, the domain of the microstructure model can thus only be on the order of a few millimeters. In contrast, some industrial process models are on the order of meters in dimension.

One approach to circumvent this problem is to develop a constitutive relationship using the results predicted by the microstructure model. In real experiments, empirical constitutive laws are developed using regression analysis. These constitutive laws are then provided to a macroscale model to predict bulk stress-strain behavior during processing. The same methodology can be applied to the results of the direct FE simulations while accounting for the effects of fraction solid, fraction porosity and grain size on constitutive behavior. Within the macroscale model, variations in these parameters can be calculated at each FE integration point. For example, the evolution of fraction solid could be obtained from the temperature, the grain size from the cooling rate and thermal gradient (e.g. [46]), and the fraction porosity from various porosity models incorporating the effects of hydrogen solubility and encapsulated liquid (e.g. [47-49]).

The FE simulations have identified four critical characteristics of semi-solid constitutive behavior, which are listed below in decreasing importance:

(a) The fraction solid;

(b) the grain size;

(c) non-classical geometric strain hardening;

(d) the porosity (which plays a minor role in semi-solid constitutive behavior, within the limits examined in this model).

Based on these characteristics, a new constitutive relationship should include a term relating the bulk constitutive behavior to fraction solid of the form shown in (1), a strain hardening term of the form shown in (2) to account for the geometric hardening and grain size effects and a term of the form shown in (3) to characterize the effect of fraction porosity on flow stress such that this effect decreases with decreasing fraction solid.

$$
\begin{aligned}
& \sigma \propto f_{\mathrm{s}} \sigma_{\mathrm{s}}, \\
& \sigma \propto \varepsilon_{\mathrm{p}}^{n}, \\
& \sigma \propto\left(1-\frac{f_{\mathrm{p}}}{1-f_{\mathrm{s}}}\right),
\end{aligned}
$$

where $\sigma_{\mathrm{s}}$ is the solid flow stress, $\varepsilon_{\mathrm{p}}$ is the plastic strain and $n$ is the strain hardening parameter.

Given the requirements of (1)-(3), the results from the semi-solid deformation simulations were compiled and used to formulate a new constitutive relationship for the aluminum alloy AA5182 in the semi-solid state, $0.75<f_{\mathrm{s}}<0.95$,

$$
\sigma=K_{\mathrm{p}}\left(f_{\mathrm{s}} \sigma_{\mathrm{s}}\right)\left(\varepsilon_{\mathrm{p}}+\varepsilon_{0}\right)^{n}\left(1-f_{\mathrm{p}} /\left(1-f_{\mathrm{s}}\right)\right),
$$

where $K_{\mathrm{p}}$ is the porosity proportionality coefficient and $\varepsilon_{0}$ is the initial strain such that $\sigma\left(\varepsilon_{\mathrm{p}}=0\right)=\sigma_{\text {yield }} . \varepsilon_{0}$ will therefore vary as a function of temperature and fraction solid.

In (4), $K_{\mathrm{p}}$ has a value of 1.0 when $f_{\mathrm{p}}=0$ and 0.87 when $f_{\mathrm{p}}>0 . \sigma_{\mathrm{s}}$ is the same as the flow stress for the solid portion of the direct FE simulations, i.e. the Ludwik equation from [33],

$$
\sigma_{\mathrm{s}}=(483.5-0.77 T) \dot{\varepsilon}^{0.205+0.00006 T},
$$


where $\sigma_{\mathrm{s}}$ has units of $\mathrm{MPa}, \dot{\varepsilon}$ is the bulk strain rate and $T$ is the temperature in units of ${ }^{\circ} \mathrm{C} . n$ can be determined based on the fact that the geometric hardening is a function of the intergranular liquid channel thickness, $h$, as discussed in section 3.1, with larger liquid channels having less geometric strain hardening. Although $h$ does not have a unique value, the equation proposed by Larouche et al [35] for equiaxed-globular grains enables calculation of an average $h$ based on $f_{\mathrm{s}}$ and $\bar{d}$,

$$
h=\bar{d}\left(1-f_{\mathrm{s}}^{1 / 3}\right) .
$$

Using (6) and regression analysis on the direct FE simulation results, the value of $n$ can be approximated via

$$
n=-6.35 \cdot 10^{-4} h^{2}+0.0202 h .
$$

Note that the coefficients for (7) were calculated for $h$ values given in $\mu \mathrm{m}$. As this equation is quadratic, it has an inflection point, which occurs at $h=15.9 \mu \mathrm{m}$. Above this value of $h, n$ is fixed at a value of zero. In the model geometry used for the analysis, this situation occurs only for $\bar{d}>225 \mu \mathrm{m}$ and $f_{\mathrm{s}}<0.80$. From a physical basis, the inflection point corresponds to the limit of liquid channel thickness whereby the geometric hardening effect is applicable. Above this value, there is simply too much liquid between the grains to allow for significant interaction between the solid.

Implementation of the results obtained using the direct FE simulations within a macroscale model requires not only the constitutive law developed above, but also a description of the semi-solid material behavior within the elastic regime. One simple way to model the elastic behavior of a randomly oriented composite is through the equal stress, or Reuss model [50],

$$
E_{\mathrm{ss}}=\left[\frac{f_{\mathrm{s}}}{E_{\mathrm{s}}}+\frac{1-f_{\mathrm{s}}}{E_{\mathrm{l}}}\right]^{-1},
$$

where $E_{\mathrm{s}}, E_{1}$ and $E_{\mathrm{ss}}$ are the elastic modulii for the solid, liquid and semi-solid material. This model was chosen since the liquid phase bears the majority of the deformation at low bulk strains. Furthermore, since there is a large difference in Poisson's ratio between a solid and a liquid, a modified value for the liquid modulus is used,

$$
E_{1} \rightarrow E_{1}^{\prime}=\frac{E_{1}}{1-v^{2}}
$$

A value of 0.5 was chosen for Poisson's ratio in order to conserve volume in the liquid.

\subsection{Comparison with simulations}

To validate the new relationship, the semi-solid behavior predicted by the constitutive law was compared with the semi-solid deformation simulations (figures 6 and 7). Figure 6 provides this comparison for two grain sizes: (a) $\bar{d}=300 \mu \mathrm{m}$ and (b) $\bar{d}=75 \mu \mathrm{m}$, in the range $0.75<f_{\mathrm{s}}<0.95$, and a bulk strain rate, $\dot{\varepsilon}$, of $0.0015 \mathrm{~s}^{-1}$ (note: the domains used in this comparison did not contain as-cast porosity). The symbols represent the results from the simulation, and the lines represent the true stresses as calculated by the constitutive relationship. These two grain sizes were chosen since they represent extremes within the model parameter space. The same conditions, but with the inclusion of porosity, is compared in figure 7.

As can be seen from figure 6 , the stresses predicted by the constitutive relationship correlate well with the direct FE simulations. In figure $6(a)(\bar{d}=300 \mu \mathrm{m})$, the constitutive relationship provides an excellent fit to the model predictions. In figure $6(b)(\bar{d}=75 \mu \mathrm{m})$, the fit is 


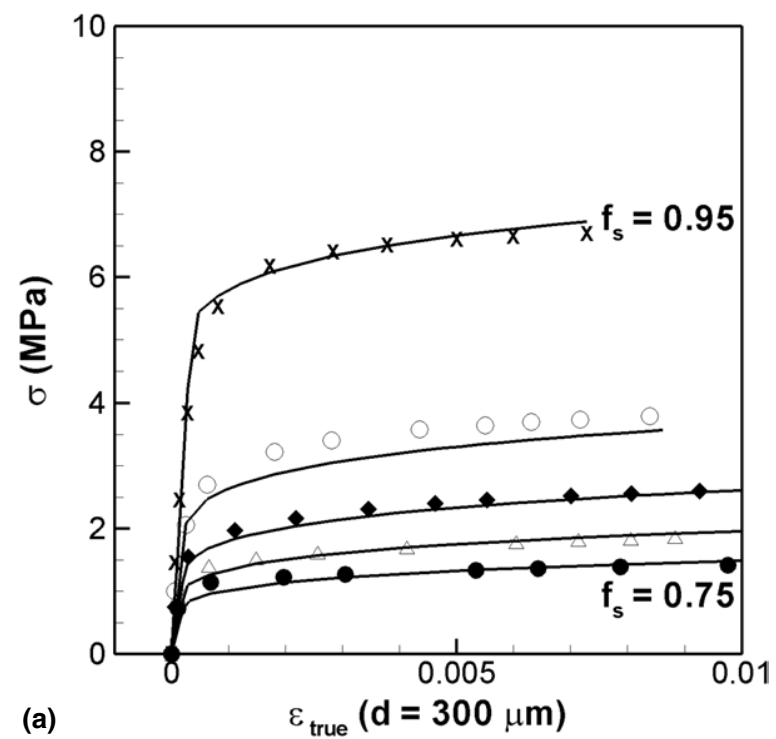

(a)

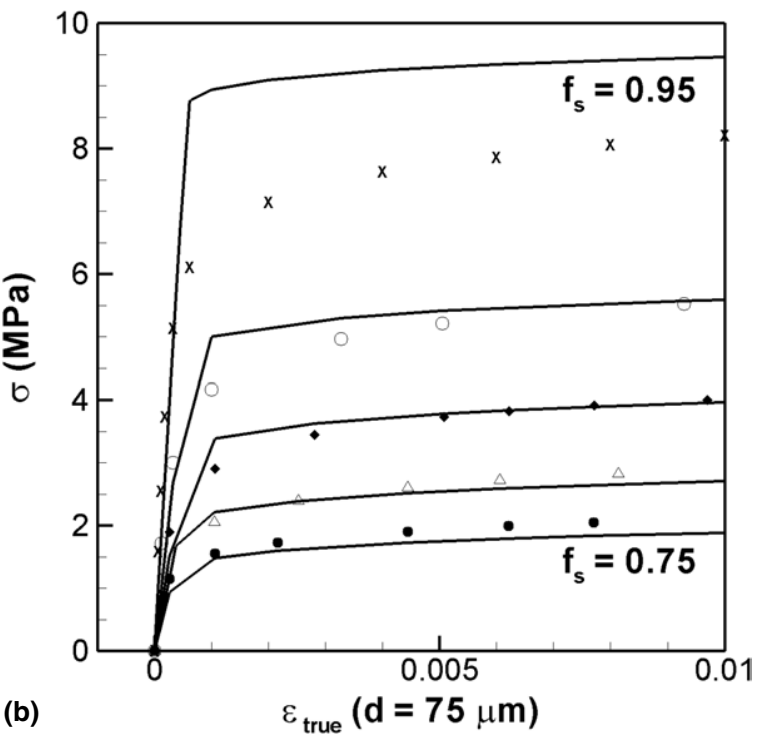

Figure 6. Validation of the constitutive relationship via the FE simulation predictions for the case without porosity: (a) $\bar{d}=300 \mathrm{~mm},(b) \bar{d}=75 \mathrm{~mm}$.

good for all but $f_{\mathrm{s}}=0.95$. For the case of $f_{\mathrm{s}}=0.95$, the true stresses predicted by the constitutive relationship are significantly higher than the simulation. This difference occurs because the constitutive relationship considers only the bulk strain rate, while the solid grains in the simulation can experience a variety of strain rates. In figure $6(b)$, the combination of small grain size and high fraction solid create very thin liquid films. Thin liquid films allow for significant strain accumulation in the solid grains, leading to a high degree of strain hardening in some of the grains (those experiencing the high strain rate). At lower fraction solid, or larger grain sizes, this effect of differential strain hardening on constitutive behavior is less of an issue because the liquid films are thicker. 


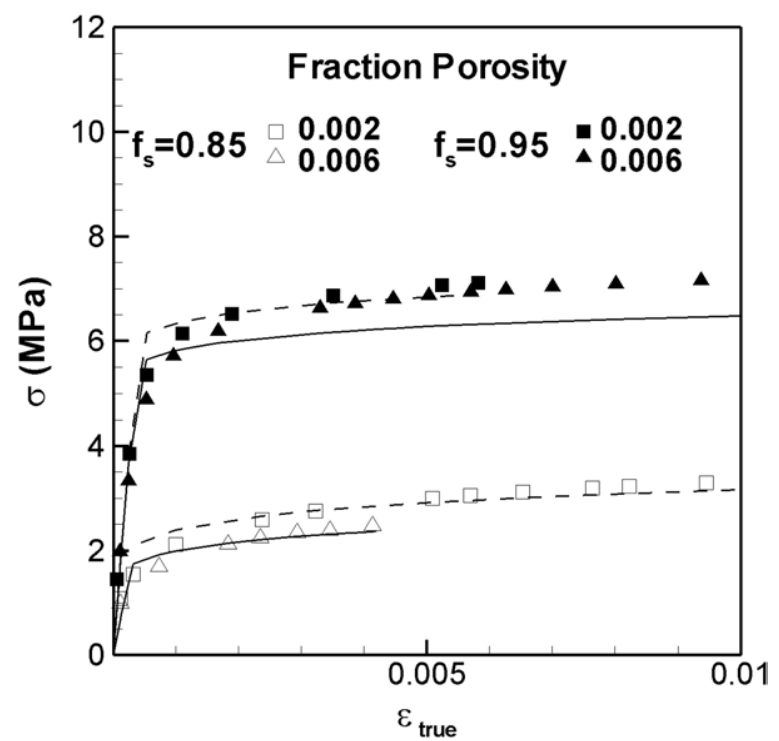

Figure 7. Validation of the constitutive relationship via the FE simulation predictions for the case where porosity is included at $f_{\mathrm{s}}=0.85$ and $f_{\mathrm{s}}=0.95(\bar{d}=150 \mu \mathrm{m})$.

As can be seen in figure 7 , the constitutive relationship also correlates well with the simulations for $f_{\mathrm{s}}=0.85$ when porosity is included. However, when porosity is included, in contrast to the trend observed in figure 6 , the constitutive relationship underestimates the flow stress at $f_{\mathrm{s}}=0.95$. The porosity term, i.e. equation (3), was the most difficult aspect of the constitutive relationship to quantify because the actual locations of individual pores have a significant effect on stress-strain response. Similar to the analysis presented in table 3 for grain geometric configuration, an analysis on 25 model runs with different porosity placement was conducted. The simulation predictions varied by $\sim 0.40 \mathrm{MPa}$ at $f_{\mathrm{s}}=0.90$, or over $10 \%$. From an application standpoint, the porosity geometric configuration with the lowest flow stresses and therefore the one that would predict the highest strains was chosen as input data for the constitutive equation.

\subsection{Comparison with experimental data}

The new constitutive relationship has been successfully validated against the microstructure model. As a final check on its accuracy, a comparison between the stresses predicted by the constitutive relationship and the experimental measurements has been made. Figure 8 shows a comparison between an experimentally obtained semi-solid stress-strain curve of hot isostatic pressing (HIP) AA5182 tested at $570{ }^{\circ} \mathrm{C}$, the FE simulation of this test, and two stress-strain curves predicted by the constitutive law $\left(f_{\mathrm{p}}=0\right.$ and $\left.f_{\mathrm{p}}=0.003\right)$. As described in [33], it is thought that the experimental fraction solid was $\sim 0.95$, and that the material was porosity-free due to the HIP processing. It should be noted that under the testing conditions, the sample exhibited very little ductility and hence a comparison can only be made under a very small range of strain.

As can be seen in figure 8, the FE simulation and the proposed constitutive relationship both reproduce the experimental behavior quite well up to the ductility limit of the test, at $\varepsilon \sim 0.00017$, which is within the elastic regime given by equation (9). It should also be noted 


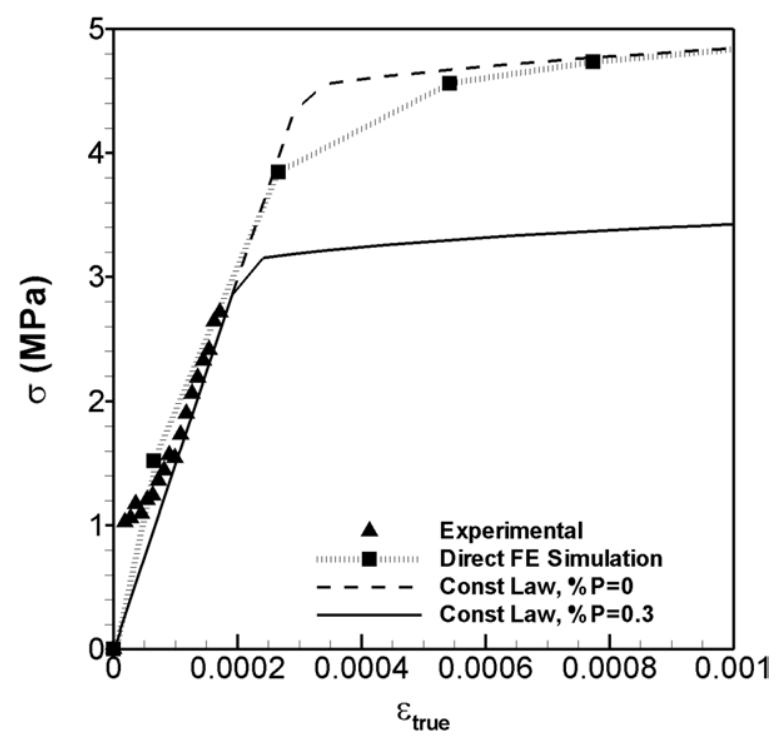

Figure 8. Comparison of the stress-strain predictions: experimental measurements [43], FE simulation and the constitutive relationship for $f_{\mathrm{p}}=0$ and $f_{\mathrm{p}}=0.003$.

that the stress-strain curves predicted by the constitutive law are also qualitatively similar to the tensile stress-strain measurements made on semi-solid material at high fraction solid [20]. The stress-strain curve based on the constitutive relationship for $f_{\mathrm{p}}=0.003$ is seen to produce a yield stress similar to the experimentally obtained failure stress, while the yield stress for the $f_{\mathrm{p}}=0$ curve is much higher. Thus, it can be hypothesized that nucleation and rapid growth of damaged inducted porosity limited the ductility leading to failure at a modest strain of $\varepsilon \sim 0.00017$ in the sample tested, but this is speculative.

In summary, the constitutive relationship of equation (4) is able to predict the stressstrain behavior once deformation of the solid grains has begun, and in a range of strain that is similar to the strain necessary for solidification cracking, or hot tear defects, to occur [51]. Prior to deformation within the solid grains, the semi-solid behavior approximates elastic-type deformation with a low modulus. In addition, and more importantly, the model provides a mechanism for incorporating the effects of grain size and porosity into a constitutive law for semi-solids. The inclusion of these microstructural phenomena is critical for using macro-scale casting models to reduce industrial defects and improve quality.

Although the constitutive law proposed in (4) is difficult to validate against experimental data, due to measurement uncertainty and reduced ductility, it can be compared qualitatively to other constitutive laws currently available in the literature. As presented in the introduction, the laws for semi-solid constitutive behavior can be divided into two general types-(a) a coupled approach in which the solid and the liquid have separate constitutive behavior [3,15-19], and (b) a single approach in which the two phases are treated as a single medium with one constitutive law [9-14]. In both cases, these relationships have generally been difficult to validate experimentally. For example, the constitutive law proposed by Fabregue et al [12] uses as a fitting parameter the fraction of grain boundary wetted liquid, a value which can be obtained from micrographs for grain-refined castings. In the case of the constitutive law proposed by Larouche et al [35], the stress is predicted to continuously increase as a function of strain whereas experimental observations [20] indicate that the semi-solid material 
reaches a plateau stress or is strain-softening. In the case of the constitutive law proposed by Ludwig [4], the model is well validated against experimental data although it does not take into account microstructural effects. As was seen in section 3.1, microstructural configuration is an important aspect to semi-solid deformation. The constitutive law developed in this work provides a basis on which future semi-solid constitutive relationships for macroscopic modeling should be based-namely taking into account the effects of microstructural features (solidification path, grain size and as-cast porosity) on the bulk semi-solid flow stress.

\section{Conclusions}

A new constitutive relationship for the semi-solid aluminum alloy AA5182 has been formulated based on a direct FE simulation of semi-solid tensile deformation using the microstructure model developed in [33]. This relationship is valid for high fraction solid, where the solid phase plays a major role in the deformation properties. Furthermore, this relationship includes the relevant effects of grain size and fraction porosity on the stress-strain predictions. In terms of their effect on constitutive behavior, fraction solid is most important, followed by grain size and then fraction porosity. These features have not previously been incorporated into a semi-solid constitutive relationship. Since it is relatively easy in a process model to calculate a local value for fraction solid, fraction porosity and grain size, the new constitutive relationship allows for the constitutive behavior to vary as a function of the solidification conditions.

\section{Acknowledgments}

The authors would like to acknowledge NSERC, EPSRC (GR/T26344) and Alcan Intl. for financial support, and thank Professor D Maijer for fruitful discussions regarding modeling of the liquid phase within the ABAQUS framework.

\section{References}

[1] Sengupta J, Cockcroft S L, Maijer D M, Wells M A and Larouche A 2004 Metall. Mater Trans. B 35523

[2] Sengupta J, Cockcroft S L, Maijer D M and Larouche A 2005 Mater. Sci. Eng. A 397157

[3] M'Hamdi M, Mo A and Martin C L 2002 Metall. Mater. Trans. A 332081

[4] Ludwig O, Drezet J M, Martin C L and Suery M 2005 Metall. Mater. Trans. A 361525

[5] Drezet J-M 1996 Direct chill and electromagnetic casting of aluminium alloys: thermomechanical effects and solidification aspects $P h D$ Ecole Polytechnique Fédérale de Lausanne

[6] Pequet C, Gremaud M and Rappaz M 2002 Metall. Mater. Trans. A 332095

[7] Suyitno, Kool W H and Katgerman L 2005 Metall. Mater Trans A 36A 1537

[8] Eskin D G, Suyitno and Katgerman L 2004 Prog. Mater. Sci. 49629

[9] van Haaften W M, Kool W H and Katgerman L 2002 Mater. Sci. Eng. A 3361

[10] Drezet J M and Eggeler G 1994 Scr. Metall. Mater. 31757

[11] Braccini M, Martin C L and Suery M 2000 Model Casting, Welding \& Adv Solidif Proces IX (Aachen, DM) ed P R Sahm et al (TMS) p 18

[12] Fabregue D, Deschamps A, Suery M and Poole W J 2006 Metall. Mater. Trans. A 37A 1459

[13] Fabregue D, Deschamps A, Suery M and Drezet J M 2006 Acta Mater. 545209

[14] Lahaie D J and Bouchard M 2001 Metall. Mater. Trans. B 32697

[15] Martin C L, Braccini M and Suery M 2002 Mater. Sci. Eng. A 325292

[16] Suery M, Martin C L, Braccini M and Brechet Y 2001 Adv. Eng. Mater. 3589

[17] Mathier V, Drezet J M and Rappaz M 2007 Modelling Simul. Mater. Sci. Eng. 15121

[18] Mathier V, Vernède S, Jarry P and Rappaz M 2009 Metall. Mater. Trans. A 40943

[19] M'Hamdi M, Mo A and Fjaer H G 2006 Metall. Mater. Trans. A 37A 3069

[20] Phillion A B, Thompson S, Cockcroft S L and Wells M A 2008 Mater. Sci. Eng. A 497388

[21] Gall K, Horstemeyer M, McDowell D L and Fan J 1999 Mech. Mater. 32277 
[22] Gao Y X, Yi J Z, Lee P D and Lindley T C 2004 Acta Mater. 525435

[23] McDowell D L, Gall K, Horstemeyer M F and Fan J 2003 Eng. Fract. Mech. 7049

[24] Horstemeyer M F, Ramaswamy S and Negrete M 2003 Mech. Mater. 35675

[25] Huber G, Brechet Y and Pardoen T 2005 Acta Mater. 532739

[26] Pardoen T and Hutchinson J W 2000 J. Mech. Phys. Solids 482467

[27] Pardoen T and Hutchinson J W 2002 Acta Mater. 51133

[28] Lassance D, Fabregue D, Delannay F and Pardoen T 2007 Prog. Mater. Sci. 5262

[29] Harnish S F, Padilla H A, Gore B E, Dantzig J A, Beaudoin A J, Robertson I M and Weiland H 2005 Metall. Mater. Trans. A 36A 357

[30] Mathier V, Jacot A and Rappaz M 2004 Modelling Simul. Mater. Sci. Eng. 12479

[31] Vernede S, Jarry P and Rappaz M 2006 Acta Mater. 544023

[32] Vernede S and Rappaz M 2007 Acta Mater. 551703

[33] Phillion A B, Cockcroft S L and Lee P D 2008 Acta Mater. 564328

[34] Clyne T W and Davies G J 1981 Br. Foundryman 7465

[35] Larouche D, Langlais J, Wu W and Bouchard M 2006 Metall. Mater. Trans. B 37B 431

[36] Thompson S, Cockcroft S L and Wells M A 2004 Mater. Sci. Technol. 20497

[37] Ludwig O, Drezet J M, Meneses P, Martin C L and Suery M 2005 Mater. Sci. Eng. A 413174

[38] Watson I G, Lee P D, Dashwood R J and Young P 2006 Metall. Mater. Trans. A 37A 551

[39] Ghosh S, Nowak Z and Lee K 1997 Acta Mater. 452215

[40] Brechet Y, Embury J D, Tao S and Luo L 1991 Acta Metall. Mater. 391781

[41] Spowart J E 2006 Mater. Sci. Eng. A 425225

[42] Venkatramani G, Ghosh S and Mills M 2007 Acta Mater. 553971

[43] Phillion A B, Cockcroft S L and Lee P D 2008 Mater. Sci. Eng. A 491237

[44] Eady J A and Smith D M 1986 Mater. Forum 9217

[45] Major J F 1998 AFS Trans. 105901

[46] Ares A E, Caram R and Schvezov C E 2003 Light Metals 2003 (San Diego, CA) ed P Crepeau (TMS) p 1055

[47] Lee P D, Chirazi A, Atwood R C and Wang W 2004 Mater. Sci. Eng. A 36557

[48] Atwood R C, Sridhar S, Zhang W and Lee P D 2000 Acta Mater. 48405

[49] Zhu J D, Cockcroft S L and Maijer D M 2006 Metall. Mater. Trans. A 37A 1075

[50] Clyne T W and Withers P J 1993 An Introduction to Metal Matrix Composites (Cambridge: Cambridge University Press)

[51] Mitchell J B, Cockcroft S L, Viano D, Davidson C J and St John D 2007 Metall. Mater. Trans. A 382503 